Andrea Bauer ${ }^{1}$

J. Geier ${ }^{2}$

H. Lessmann ${ }^{2}$

P. Elsner ${ }^{1}$

\section{Kontaktallergien gegen Textilfarbstoffe. Ergebnisse des Informationsverbundes Dermatologischer Kliniken (IVDK)}

\author{
Contact Allergy to Textile Dyes. Results of the Information Network of \\ Departments of Dermatology (IVDK)
}

\section{Zusammenfassung}

In den Jahren 1998 bis 2002 wurden in den dem IVDK angeschlossenen dermatologischen Abteilungen 1137 Patienten wegen des Verdachtes auf eine Textilallergie epikutan getestet. Bei 696 dieser Patienten wurde die DKG-Testreihe „Textilfarbstoffe“ überprüft. Dabei ergaben sich am häufigsten allergische Reaktionen auf p-Aminoazobenzol (9,1\% positive Reaktionen), gefolgt von Dispers Blau 106/124 (7,7\%) und Dispers Orange 3 (3,4\%). Die Quote positiver Reaktionen auf das in der Standardreihe enthaltene p-Phenylendiamin betrug 4,3\%. Während die Konkordanz positiver Reaktionen auf p-Phenylendiamin und p-Aminoazobenzol deutlich war (Cohen's kappa $=0,67$ ), ergab die Untersuchung der Konkordanz von p-Aminoazobenzol bzw. p-Phenylendiamin einerseits und den in der Testreihe enthaltenen Azofarbstoffen andererseits lediglich für die Kombination p-Aminoazobenzol/Dispers Orange 3 eine substanzielle Übereinstimmung (kappa $=0,69$ ), die sich durch deren enge chemische Verwandtschaft erklärt. Zwischen p-Phenylendiamin und Dispers Orange 3 bestand eine mäßige Konkordanz (kappa =0,40). Die

\section{Abstract}

Between 1998 and 2002, 1137 patients were tested for textile allergy in the participating centres of the IVDK. In 696 of these patients, the textile dye series of the German Contact Dermatitis Research Group (DKG) has been tested. Most frequently, allergic reactions to $\mathrm{p}$-aminoazobenzene ( $9.1 \%$ positive reactions) occurred, followed by disperse blue 106/124 (7.7\%) and disperse orange 3 (3.4\%). Positive reactions to p-phenylenediamine, which was tested in the standard series, were noted in $4.3 \%$ of the tested patients. The agreement of positive reactions to $\mathrm{p}$-phenylenediamine and p-aminoazobenzene was substantial (Cohen's kappa $=0.67$ ). Further analysis of concomitant positive reactions to p-phenylenediamine or p-aminoazobenzene on the one hand and the azo dyes contained in the test series on the other hand revealed substantial agreement only for the combination p-aminoazobenzene/disperse orange 3 (kappa $=0.69$ ), which can be explained by their close chemical relationship. A moderate agreement was seen with p-phenylenediamine and disperse orange $3($ kappa $=0.40)$. The test substances p-phenylenediami-

Lübeck (J. Grabbe), Mainz (D. Becker), Mannheim (C. Bayerl), Marburg (I. Effendy, H. Löffler), München

Schwabing (M. Agathos), München TU (J. Rakoski), Nürnberg (I. Müller), Osnabrück (W. Uter, S. John,

H. J. Schwanitz, N. Schürer), Rostock (H. Heise), Tübingen (G. Lischka), Ulm Univ.-Hautklinik und BWK

(H. Gall†, P. Gottlöber, G. Staib, H. Pillekamp, R. Hinrichs), Wuppertal (O. Mainusch, J. Raguz, B. Dierbach), Würzburg (J. Arnold, A. Trautmann). 
alleinige Testung von p-Aminoazobenzol oder p-Phenylendiamin als Marker für eine Sensibilisierung gegen die verschiedenen in Textilien verwendeten Azofarbstoffe ist also nicht ausreichend. Bei entsprechendem Verdacht müssen die einzelnen Farbstoffe zusätzlich überprüft werden. ne and p-aminoazobenzene were proven to be poor screening agents for most of the azo dyes. Therefore, the individual dyes have to be used for patch testing in cases of suspected textile dye allergy.

\section{Einleitung}

Kontaktallergien gegen Textilfarben sind im Vergleich zu ihrer weiten Verbreitung eher selten. Hatch und Maibach fassten Prävalenzstudien bei Epikutantestpatienten zusammen, wobei in Abhängigkeit vom Patientengut die Sensibilisierungsquoten gegen Textilfarben zwischen 0 und 5,8\% der konsekutiv Getesteten lagen [1]. Am häufigsten werden Typ-IV-Sensibilisierungen gegen Dispersionsfarbstoffe beschrieben, die für die Färbung von synthetischen und gemischten Fasern verwendet werden [1 -4]. Weitaus seltener sind Sensibilisierungen gegen Nicht-Dispersionsfarbstoffe $[5,6]$.

Durch Textilfarbenallergien ausgelöste Kontaktekzeme manifestieren sich meist in den großen Beugen, im Nacken und an den Oberschenkeln, also an den Körperstellen, die in besonders intensiven Kontakt mit der Bekleidung kommen [3,7-11].

Ziel der vorliegenden Studie war es, das Allergenspektrum und insbesondere die Häufigkeit von Kontaktallergien gegen Textilfarben bei Patienten, die unter dem Verdacht auf eine Textilallergie epikutan getestet wurden, zu identifizieren. Darüber hinaus wurde die Tauglichkeit von p-Phenylendiamin und p-Aminoazobenzol als Indikatorallergene bei Textilfarbenallergie überprüft.

\section{Material und Methoden}

Für die vorliegende Arbeit wurden die Daten von 46250 Patienten ausgewertet, die in 37 dem IVDK [12, www.ivdk.org] angeschlossenen dermatologischen Abteilungen in den Jahren 1998 bis 2002 getestet wurden. Bei 1137 dieser Patienten (2,5\%) wurde die Epikutantestung unter dem Verdacht auf eine Kontaktallergie gegen Textilien vorgenommen. Die Daten dieser Patienten wurden im Vergleich zu denen der übrigen 45113 Patienten (Kontrollgruppe) analysiert.

Die Durchführung der Epikutantestungen und die Dokumentation der Testreaktionen erfolgten gemäß den Leitlinien der Deutschen Kontaktallergie-Gruppe (DKG) [13]. Die Expositionsdauer des Epikutantests betrug 48 Stunden bei 30648 Patienten (66,3\% der Getesteten) und 24 Stunden bei 15602 Patienten (33,7\%). Die Testsubstanzen wurden im Allgemeinen von der Firma Hermal, Reinbek, beschafft. Lediglich in einer Klinik (276 Patienten) wurde für die Standardreihe der TRUE-Test ${ }^{\circledR}$, Pharmacia \& Upjohn, Hilleröd, Dänemark, eingesetzt. Die Testsubstanzen „Dispers Blau 106“ und „Dispers Blau 124“ stammten von Chemotechnique, Malmö, Schweden.
Die statistische Auswertung erfolgte in der Zentrale des IVDK an der Universität Göttingen unter Verwendung des Programmpaketes SAS, Version 8.02 (SAS Institute, Cary, NC, USA). Alle Auswertungen basieren auf den Testreaktionen an Tag 3. Die Berechnung alters- und geschlechtsstandardisierter Reaktionsquoten erfolgte nach den Kriterien von PAFS [14]. Häufigkeitsunterschiede bestimmter Merkmale in den disjunkten Subgruppen wurden mit dem exakten Test von Fisher auf statistische Signifikanz geprüft, wobei ein p-Wert von $<0,05$ als Signifikanzgrenze angenommen wurde. Die Quantifizierung der Assoziation bestimmter Merkmale erfolgte durch die Berechnung von Cohen's kappa.

\section{Ergebnisse}

Im Laufe der Jahre 1998 bis 2002 variierte der Anteil von Patienten, die wegen des Verdachtes auf eine Textilallergie getestet wurden, zwischen 2,2 und 2,7\%, ohne dass dabei eine Tendenz zur Zu- oder Abnahme erkennbar war.

Eine Beschreibung der Testpopulation und der Kontrollgruppe mithilfe des MOAHLFA-Indexes [14] ist in Tab.1 wiedergegeben. Außerdem ist dort angegeben, wie viele Patienten in beiden Gruppen an Ulcus cruris bzw. Stauungsdermatitis bei chronischvenöser Insuffizienz (CVI) und wie viele an Fußekzem litten. Aufgrund des großen Umfanges der Stichproben unterscheiden sich die Häufigkeiten sämtlicher hier aufgeführter Merkmale signifikant. Am auffälligsten ist der Unterschied in der Altersstruktur; der Anteil von Patienten im Alter von 40 Jahren und mehr ist in der Gruppe mit Verdacht auf Textilallergie deutlich geringer (56,3 vs. $63,7 \%$ ). Dass die Anteile von Patienten mit Berufsder-

Tab. 1 MOAHLFA-Index sowie Häufigkeit von Ulcus cruris bzw. Stauungsdermatitis bei chronisch-venöser Insuffizienz (CVI) und Häufigkeit von Fußekzemen bei Pat. mit bzw. ohne V.a. Textilallergie

\begin{tabular}{|c|c|c|c|c|c|}
\hline & & \multicolumn{2}{|c|}{$\begin{array}{l}\text { V.a. Textilallergie } \\
(n=1137)\end{array}$} & \multicolumn{2}{|c|}{$\begin{array}{l}\text { andere } \\
(n=45113)\end{array}$} \\
\hline & & Anzahl & Prozent & Anzahl & Prozent \\
\hline männlich & M & 471 & 41,4 & 16993 & 37,7 \\
\hline Berufsdermatose & $\mathrm{O}$ & 110 & 9,7 & 6241 & 13,8 \\
\hline atopische Dermatitis & $A$ & 213 & 18,7 & 7340 & 16,3 \\
\hline Hand-Dermatitis & $\mathrm{H}$ & 220 & 19,3 & 11842 & 26,2 \\
\hline Bein-Dermatitis & $\mathrm{L}$ & 136 & 12,0 & 4573 & 10,1 \\
\hline Gesichts-Dermatitis & $\mathrm{F}$ & 85 & 7,5 & 6329 & 14,0 \\
\hline Alter mind. 40 Jahre & A & 640 & 56,3 & 28727 & 63,7 \\
\hline Ulcus cruris / CVI & & 28 & 2,5 & 3205 & 7,1 \\
\hline Fußekzem & & 77 & 6,8 & 1116 & 2,5 \\
\hline
\end{tabular}


Tab. 2696 Pat. mit V.a. Textilallergie, Reaktionen auf die DKG-Testreihe „Textilfarben“ (Nr. 24) und p-Phenylendiamin

\begin{tabular}{|c|c|c|c|c|c|c|c|c|c|c|c|}
\hline Substanz & $\begin{array}{l}\text { Azo- } \\
\text { Farbstoff? }\end{array}$ & $\begin{array}{l}\text { Testkonzentration } \\
\text { (in Vaseline) }\end{array}$ & $\begin{array}{l}\text { Anzahl } \\
\text { Getestete }\end{array}$ & $\begin{array}{l}\text { Reak } \\
\text { neg }\end{array}$ & $\begin{array}{l}\text { ionen } \\
?\end{array}$ & $\begin{array}{l}\text { In } T c \\
f\end{array}$ & + & ++ & +++ & ir & $\begin{array}{l}\% \text { positive } \\
\text { Reaktionen }\end{array}$ \\
\hline p-Aminoazobenzol (Cl 11000) & & $1,00 \%$ & 243 & 216 & 4 & 0 & 11 & 10 & 1 & 1 & 9,1 \\
\hline Dispers Blau 106/124 & ja & $1,00 \%$ & 659 & 594 & 13 & 0 & 21 & 20 & 10 & 1 & 7,7 \\
\hline Dispers Blau 106 & ja & $1,00 \%$ & 263 & 238 & 4 & 1 & 11 & 5 & 3 & 1 & 7,2 \\
\hline Dispers Blau 124 & ja & $1,00 \%$ & 263 & 242 & 3 & 0 & 9 & 5 & 3 & 1 & 6,5 \\
\hline Dispers Orange 3 (Cl 11 005) & ja & $1,00 \%$ & 680 & 652 & 5 & 0 & 12 & 8 & 3 & 0 & 3,4 \\
\hline Dispers Rot 17 (Cl 11210) & ja & $1,00 \%$ & 680 & 654 & 11 & 1 & 9 & 3 & 1 & 1 & 1,9 \\
\hline Dispers Blau 1 & & $1,00 \%$ & 530 & 518 & 2 & 0 & 6 & 2 & 1 & 1 & 1,7 \\
\hline Dispers Gelb 3 (Cl 11855) & ja & $1,00 \%$ & 679 & 665 & 4 & 1 & 8 & 1 & 0 & 0 & 1,3 \\
\hline Dispers Rot 1 (Cl 11110) & ja & $1,00 \%$ & 680 & 660 & 9 & 1 & 5 & 4 & 0 & 1 & 1,3 \\
\hline Acid Yellow 36 (Cl 13065) & ja & $1,00 \%$ & 186 & 183 & 1 & 0 & 2 & 0 & 0 & 0 & 1,1 \\
\hline Bismark Brown R & ja & $0,50 \%$ & 186 & 183 & 0 & 0 & 1 & 0 & 1 & 1 & 1,1 \\
\hline Dispers Rot 11 (Cl 62 015) & & $1,00 \%$ & 121 & 120 & 0 & 0 & 0 & 0 & 1 & 0 & 0,8 \\
\hline Dispers Gelb 9 (Cl 10375) & & $1,00 \%$ & 195 & 193 & 1 & 0 & 0 & 0 & 1 & 0 & 0,5 \\
\hline Dispers Blau 3 (Cl 61505) & & $1,00 \%$ & 680 & 676 & 1 & 0 & 3 & 0 & 0 & 0 & 0,4 \\
\hline Naphthol AS (Cl 37505$)$ & & $1,00 \%$ & 681 & 676 & 2 & 0 & 2 & 1 & 0 & 0 & 0,4 \\
\hline p-Phenylendiamin (Cl 76060) & & $1,00 \%$ & 644 & 605 & 10 & 0 & 17 & 7 & 4 & 1 & 4,3 \\
\hline
\end{tabular}

matosen, Handekzem oder Gesichtsekzem ebenfalls reduziert sind, ist auf die spezielle Testindikation „V.a. Textilallergie“ in dieser Gruppe zurückzuführen. Die differenzierte Betrachtung der Tabelle zeigt, dass die erhöhte Quote an Beinekzemen bei den Patienten mit Textildermatitis wie eingangs erwähnt auf den intensiven Hautkontakt mit den Textilien im Bereich der Oberschenkel zurückzuführen ist, und nicht etwa auf einen höheren Anteil von Patienten mit CVI.

Beim Vergleich der so genannten „Allergen-Hitlisten“, also der Reaktionsquoten auf die häufigsten Allergene, ergeben sich bei den Patienten mit Verdacht auf Textilallergie erhöhte Reaktionsquoten auf zwei Allergene, nämlich Kaliumdichromat (unadjustiert 6,6 vs. $4,4 \%$ positive Reaktionen; alters- und geschlechtsstandardisiert 6,5 vs. $4,2 \%$ ) und p-Phenylendiamin (unadjustiert 4,8 vs. 4,2\%; alters- und geschlechtsstandardisiert 4,7 vs. $4,1 \%$ ). Es ist jedoch nur der Unterschied bei Kaliumdichromat statistisch signifikant $(p<0,0001)$. Auf drei Allergene ergaben sich bei den Patienten mit Verdacht auf Textilallergie erniedrigte Reaktionsquoten (sowohl unadjustiert als auch alters- und geschlechtsstandardisiert). Dies waren: Perubalsam (standardisiert 7,8 vs. 9,3\%), Wollwachsalkohole (standardisiert 2,6 vs. 4,4\%) und Amerchol L 101 (standardisiert 3,0 vs. 5,4\%). Bei allen übrigen Allergenen der Standardreihe differierten sowohl die unadjustierten als auch die alters- und geschlechtsstandardisierten Reaktionsquoten lediglich im Bereich von unter einem Prozent (Daten nicht im Detail gezeigt).

Die DKG-Testreihe „Textilfarbstoffe“ wurde im Untersuchungszeitraum dreimal in ihrer Zusammensetzung geändert. Im Juni 1999 wurden die einzelnen Testsubstanzen Dispers Blau 106 und Dispers Blau 124 aus der Testreihe entfernt; die Testungen mit dem Mix aus beiden Farbstoffen („Dispersionsblau-Mix 124/106“ von Hermal, hier bezeichnet als „Dispers Blau 106/124“) wurden fortgeführt. Im Dezember 2001 wurde die Reihe um einige Farbstoffe erweitert, so dass ein größeres Spekt- rum von Textil- und Lederfarbstoffen abgedeckt wurde. Diesem Umstand wurde auch durch eine Namensänderung Rechnung getragen.

Insgesamt wurde die Testreihe bei 696 der 1137 Patienten mit Verdacht auf Textilallergie überprüft. Die Testergebnisse sind in Tab. 2 zusammengestellt. In dieser Tabelle sind außerdem die Testreaktionen auf p-Phenylendiamin aufgeführt, die bei den mit dieser DKG-Testreihe getesteten Patienten beobachtet wurden. Am häufigsten ergaben sich Reaktionen auf p-Aminoazobenzol (9,1\%), gefolgt von Dispers Blau 106/124 (7,7\%). Die beiden einzelnen Bestandteile dieses Mixes führten mit ähnlicher Häufigkeit zu positiven Reaktionen (7,2 bzw. 6,5\%). Dispers Orange 3, das während des gesamten Untersuchungszeitraumes in der Testreihe enthalten war, löste bei 3,4\% der Getesteten allergische Reaktionen aus. Alle anderen Farbstoffe führten bei weniger als $2 \%$ der Patienten zu positiven Reaktionen.

Bei etlichen der in dieser DKG-Testreihe enthaltenen Substanzen handelt es sich um Azofarbstoffe, also um so genannte „ParaStoffe" im weitesten Sinne. Die Substanz p-Aminoazobenzol, die selbst nicht als Farbstoff eingesetzt wird, wird bei der Epikutantestung als Marker für eine Sensibilisierung gegen Azofarbstoffe angesehen. Anhand der vorliegenden Daten wurde überprüft, wie häufig positive Reaktionen auf die in der Testreihe enthaltenen Azofarbstoffe mit positiven Reaktionen auf p-Aminoazobenzol korrelieren. Außerdem wurde untersucht, wie häufig Konkordanzen zwischen positiven Reaktionen auf das in der Standardreihe enthaltene p-Phenylendiamin und die in der Textilfarbenreihe enthaltenen Azofarbstoffe auftreten. Das Ergebnis dieser Analyse ist in Tab. 3 dargestellt. Lediglich zwischen p-Aminoazobenzol und Dispers Orange 3 ergibt sich eine substanzielle Übereinstimmung (kappa $=0,69$ ). Zwischen p-Phenylendiamin und Dispers Orange 3 besteht eine mäßige Konkordanz (kap$\mathrm{pa}=0,40$ ). Für zwei weitere Paarungen konnten geringe Konkordanzen festgestellt werden, nämlich: p-Aminoazobenzol/Dis- 
Tab. 3 Konkordanz zwischen positiven Reaktionen auf Azofarbstoffe einerseits und p-Phenylendiamin bzw. p-Aminoazobenzol andererseits bei 696 Pat. mit V.a. Textil-Allergie, bei denen die DKG-Testreihe „Textilfarben“ (Nr. 24) überprüft wurde, quantifiziert mit Cohen's kappa, jeweils mit 95\%-Konfidenzintervall

\begin{tabular}{|lcc|}
\hline Azofarbstoff & $\boldsymbol{p}$-Phenylendiamin & $\boldsymbol{p}$-Aminoazobenzol \\
\hline Dispers Blau 106/124 & $0,00[-0,08-0,07]$ & $0,00[-0,12-0,12]$ \\
\hline Dispers Blau 106 & $-0,05[-0,08-0,03]$ & $-0,10[-0,16-0,03]$ \\
\hline Dispers Blau 124 & $-0,05[-0,07-0,03]$ & $-0,10[-0,16-0,03]$ \\
\hline Dispers Orange 3(Cl 11005) & $0,40[0,21-0,59]$ & $0,69[0,50-0,87]$ \\
\hline Dispers Rot 17 (Cl 11210) & $0,03[-0,07-0,13]$ & $0,19[-0,02-0,34]$ \\
\hline Dispers Gelb 3(Cl 11855) & $-0,02[-0,03-0,01]$ & $0,06[-0,08-0,21]$ \\
\hline Dispers Rot 1 (Cl 11 110) & $0,10[-0,05-0,24]$ & $0,06[-0,08-0,21]$ \\
\hline Acid Yellow 36 (Cl 13065) & $0,15[-0,13-0,43]$ & $0,10[-0,10-0,30]$ \\
\hline Bismark Brown R & $-0,02[-0,04-0,00]$ & $0,10[-0,10-0,30]$ \\
\hline
\end{tabular}

pers Rot 17 (kappa = 0,19), p-Phenylendiamin/Acid Yellow 36 (kappa $=0,15$ ). In allen übrigen Fällen war praktisch keine Übereinstimmung vorhanden. Dagegen war die Konkordanz positiver Reaktionen auf p-Phenylendiamin und p-Aminoazobenzol sehr deutlich (Cohen's kappa 0,67; 95\% Konfidenzintervall $0,47-0,87)$.

\section{Diskussion}

In den Jahren 1998 bis 2002 betrug der Anteil von Patienten mit Verdacht auf Textilallergie unter den insgesamt im IVDK erfassten Patienten mit gewissen Schwankungen konstant 2,5\%. Eine Tendenz zur Zunahme ist in dem hier untersuchten Datenmaterial nicht zu erkennen. Die Häufigkeit dieser Testindikation liegt damit - soweit aufgrund unterschiedlicher methodischer Ansätze ein Vergleich möglich ist - im Bereich dessen, was aus anderen europäischen Ländern berichtet wird [1].

In Übereinstimmung mit anderen Untersuchungen [11] führten Dispers Blau 106 und Dispers Blau 124 unter den getesteten Textilfarbstoffen am häufigsten zu allergischen Reaktionen. Die Substanzen haben ein hohes Sensibilisierungspotenzial, weisen große chemische Ähnlichkeit auf $[8,15,16]$, und sind schwer voneinander zu trennen. Dispers Blau 124 ist wahrscheinlich stets mit Dispers Blau 106 verunreinigt; Zweifel an der Reinheit der einzelnen Testsubstanzen sind daher berechtigt. Aus diesem Grund entschloss sich die DKG 1999, auf die Testung der beiden einzelnen Farbstoffe zu verzichten. Wie eine frühere Datenanalyse des IVDK ergab, besteht eine hohe Konkordanz positiver Reaktionen auf beide Farbstoffe untereinander und mit dem Mix „Dispers Blau 106/124“ [8]. Die Testung des Mixes führte auch in dieser Untersuchung mit ähnlicher Häufigkeit zu allergischen Reaktionen wie die Testung der beiden einzelnen Farbstoffe. Sensibilisierungen gegen diese Textilfarbstoffe sind so häufig, dass die DKG Dispers Blau 106/124 im November 2001 in die Standardreihe übernommen hat. Nach aktuellen Auswertungen von IVDK-Daten ergibt sich dabei eine Quote von 1,3\% positiven Reaktionen [17].
Auch auf die meisten der übrigen in der entsprechenden DKG-Testreihe enthaltenen Farbstoffe waren bei mehr als 1\% der Getesteten allergische Reaktionen festzustellen. Lediglich für Dispers Rot 11, Dispers Gelb 9, Dispers Blau 3 und Naphthol AS lagen die Reaktionsquoten niedriger. Sofern sich dies auf längere Sicht bestätigt, wäre zu überlegen, ob der Verbleib dieser Substanzen in einer Textilfarbstoffreihe sinnvoll ist.

Die Auswertung der Konkordanz zwischen allergischen Reaktionen auf Azofarbstoffe und p-Aminoazobenzol zeigt, dass bis auf eine Ausnahme die Testung von p-Aminoazobenzol als Marker für eine Sensibilisierung gegen die in dieser Testreihe enthaltenen Azofarbstoffe nicht geeignet ist. Die höhere Konkordanz positiver Reaktionen zwischen p-Aminoazobenzol und Dispers Orange 3 ist darauf zurückzuführen, dass hier eine sehr enge chemisch-strukturelle Verwandtschaft vorliegt $[18,19]$. In allen übrigen Fällen kann die Epikutantestung der einzelnen Farbstoffe nicht durch eine Überprüfung von p-Aminoazobenzol ersetzt werden. Aus früheren Analysen von IVDK-Daten ist eine hohe Konkordanz positiver Reaktionen auf p-Phenylendiamin und p-Aminoazobenzol bekannt $[18,19]$. Da zudem aktive Sensibilisierungen durch den Epikutantest mit p-Aminoazobenzol beschrieben worden sind [20], stellt sich die Frage, ob diese Substanz angesichts der geringen diagnostischen Aussage nicht aus dieser Testreihe entfernt werden sollte.

Die erhöhte Sensibilisierungsquote gegenüber Dichromat in der Gruppe der Patienten mit Verdacht auf Textildermatitis ist nicht ohne Weiteres zu erklären. Chromathaltige Farben werden sicher nicht in Textilien eingesetzt. Aus einer früheren IVDK-Datenanalyse ist aber bekannt, dass bei Frauen mit Dichromatallergie gehäuft Fußekzeme zu finden sind, und bei diesen wiederum häufig positive Reaktionen auf Dispersionsfarbstoffe beobachtet wurden [21]. Da auch in der hier untersuchten Gruppe von Patienten mit Verdacht auf Textilallergie eine ähnliche Konstellation vorliegt, könnte eine Erklärung für die Häufung von Dichromatsensibilisierungen sein, dass bei einem Teil dieser Patienten primär Schuhekzeme vorlagen, ausgelöst durch Dichromat und Farbstoffe im Leder, und sekundär eine Textilunverträglichkeit bei bestehender Farbstoffallergie hinzukam. Eine alternative Erklärungsmöglichkeit wäre, dass diese Gruppe von Patienten ganz allgemein leichter allergisch gegen Substanzen reagiert, mit denen die Haut ständig in Berührung ist, also gegen Kleidung und Schuhe.

Bei Perubalsam, Wollwachsalkoholen und Amerchol L 101 handelt es sich um typische Allergene von Patienten mit Stauungsekzem oder Ulcus cruris bei CVI [22,23]. Da solche Patienten unter denjenigen, die wegen des Verdachts auf eine Textilallergie getestet wurden, deutlich seltener vertreten sind, ist die Reaktionsquote auf diese drei Stoffe ebenfalls vermindert. Die Unterschiede in der Reaktionshäufigkeit stehen also nicht direkt mit der Frage der Textilallergie in Zusammenhang.

\section{Schlussfolgerungen}

Die Textilfarben Dispers Blau 106/124 und Dispers Orange 3 führten bei den hier untersuchten Patienten mit Verdacht auf Textilallergie am häufigsten zu allergischen Reaktionen. Die al- 
leinige Testung von p-Phenylendiamin oder p-Aminoazobenzol als Indikator für eine Sensibilisierung gegen die verschiedenen in Textilien verwendeten Azofarbstoffe ist nicht ausreichend. Bei entsprechendem Verdacht müssen die einzelnen Farbstoffe getestet werden.

\section{Literatur}

${ }^{1}$ Hatch KL, Maibach HI. Textile dye allergic contact dermatitis prevalence. Contact Dermatitis 2000; 42: 187-195

2 Elsner P. Allergische und irritative Textildermatitis. Schweiz Med Wschr 1994; 124: 111-118

${ }^{3}$ Seidenari S, Manzini BM, Danese P. Contact sensitization to textile dyes: description of 100 subjects. Contact Dermatitis 1991; 24: 253 - 258

${ }^{4}$ Hatch KL, Maibach HI. Textile dye dermatitis. J Am Acad Dermatol 1995; 32: 631 - 639

${ }^{5}$ Manzini BM, Motolese A, Conti A, Ferdani G, Seidenari S. Sensitization to reactive textile dyes in patients with contact dermatitis. Contact Dermatitis 1996; 34: 172 - 175

${ }^{6}$ Seidenari S, Manzini BM, Schiavi ME, Motolese A. Prevalence of contact allergy to non-dispers azo dyes for natural fibres: a study in 1814 consecutive patients. Contact Dermatitis 1995; 33: 118-122

7 Dooms-Goossens A. Textile dye dermatitis. Contact Dermatitis 1992; 27: $321-323$

${ }^{8}$ Uter W, Geier J, Lessmann H, Hausen BM. Contact allergy to Disperse Blue 106 and Disperse Blue 124 in German and Austrian patients, 1995 to 1999. Contact Dermatitis 2001; 44: $173-177$

${ }^{9}$ Lodi A, Ambonati M, Coassini A, Chiarlli G, Mancini L, Crosti C. Textile dye contact dermatitis in an allergic population. Contact Dermatitis 1998; 39: $314-315$

${ }^{10}$ Pratt M, Taraska V. Disperse blue dyes 106 and 124 are common causes of textile dermatitis and should serve as screening allergens for this condition. Am J Contact Dermatitis 2000; 11: 30-41

${ }^{11}$ Seidenari S, Giusti F, Massone F, Mantovani L. Sensitization to disperse dyes in a patch test population over a five-year period. Am J Contact Dermatitis 2002; 13: $101-107$
${ }^{12}$ Schnuch A, Geier J, Uter W. Der Informationsverbund Dermatologischer Kliniken (IVDK). Klinische Epidemiologie zur Prävention des Allergischen Kontaktekzems. Hautarzt 2001; 52: 582 - 584

${ }^{13}$ Schnuch A, Aberer W, Agathos M, Brasch J, Frosch PJ, Fuchs Th, Richter G. Für die Deutsche Kontaktallergie-Gruppe: Leitlinien der Deutschen Dermatologischen Gesellschaft (DDG) zur Durchführung des Epikutantests mit Kontaktallergenen. Hautarzt 2001; 52: 864-866

${ }^{14}$ Schnuch A, Geier J, Uter W, Frosch PJ et al. National rates and regional differences in sensitization to allergens of the standard series. Population-adjusted frequencies of sensitization (PAFS) in 40,000 patients from a multicenter study (IVDK). Contact Dermatitis 1997; 37: 200-209

15 Hausen BM, Brinkmann J, Dohn W. Lexikon der Kontaktallergene. Landsberg: ecomed Verlag, 1992

${ }^{16}$ Menezes Brandao F, Hausen BM. Cross reaction between Disperse Blue dyes 106 and 124. Contact Dermatitis 1987; 16: 289-290

${ }^{17}$ Uter W, Geier J, Hausen BM. Contact allergy to Disperse Blue 106/124 mix in consecutive German, Austrian and Swiss patients. Contact Dermatitis 2003; 48: 286-287

18 Uter W, Lessmann H, Geier J, Becker D, Fuchs Th, Richter G. The spectrum of allergic (cross-) sensitivity in clinical patch testing with „para amino“ compounds. Allergy 2002; 57: 319-322

19 Uter W, Lessmann H, Geier J, Becker D, Fuchs Th, Richter G. Die Epikutantestung mit „Parastoffen“. Dermatologie in Beruf und Umwelt 2002; 50: $97-104$

${ }^{20}$ Arnold WP, van Joost T, van der Valk PGM. Adding p-aminoazobenzene may increase the sensitivity of the European standard series in detecting contact allergy to dyes, but carries the risk of active sensitization. Contact Dermatitis 1995; 33: 444

${ }^{21}$ Geier J, Schnuch A, Frosch PJ. Contact allergy to dichromate in women. Dermatologie in Beruf und Umwelt 2000; 48: 4-10

${ }^{22}$ Lange-Ionescu S, Pilz B, Geier J, Frosch PJ. Kontaktallergien bei Patienten mit Stauungsdermatitis oder Ekzem der Beine. Dermatosen in Beruf und Umwelt 1996; 44: 14-22

${ }^{23}$ John SM, Geier J. Unterschenkelekzem bei chronisch-venöser Insuffizienz. Aktuelle allergologische Aspekte. Allergenspektrum bei „Beinpatienten“ im IVDK 1994-1995. Zeitschrift für Dermatologie 1998; 184: $16-23$ 